\title{
CLDN18.2 Positive
}

National Cancer Institute

\section{Source}

National Cancer Institute. CLDN18.2 Positive. NCI Thesaurus. Code C153150.

An indication that expression of Claudin-18 Isoform A2 has been detected in a sample. 